\title{
Influences upon pediatricians' willingness to refer patients to clinical research
}

\author{
This article was published in the following Dove Press journal: \\ Open Access Journal of Clinical Trials \\ 27 March 2010 \\ Number of times this article has been viewed
}

\author{
Jeanne Dalen' \\ Robert D Annett ${ }^{2}$ \\ Janet L Brody' \\ Mandy L Perryman ${ }^{3}$ \\ 'Center for Family and Adolescent \\ Research, Oregon Research Institute, \\ Portland, OR, USA; ' University of \\ New Mexico Health Sciences Center, \\ Albuquerque, NM, USA; ${ }^{3}$ School of \\ Education and Human Development, \\ Lynchburg College, Lynchburg, UA, \\ USA
}

Purpose: The purpose of this brief report is to determine factors that influence the willingness of pediatricians to refer their patients to clinical research and to explore the relationship between pediatrician characteristics and self-reported number of patients referred to clinical research.

Method: Forty-three pediatricians from an academic pediatrics department of a university children's hospital in Albuquerque, New Mexico rated how influential 10 reasons would be in their decision to refer a patient to pediatric clinical research.

Results: Differences among the influences for pediatrician referral to research were observed. The most influential consideration for referral was the scientific merit of the study, followed by patient benefit. Contextual factors and physician compensation were identified as the least important reasons pediatricians refer patients to research. Analyses also revealed significant relationships between referrals made and percentage of time spent in research activities.

Conclusions: Pediatricians may be more likely to refer their patients to clinical research studies when they believe the purpose of the study is meaningful to patients as well as to future patient populations. In addition, characteristics of the individual pediatricians may play an important role in actual referral behavior.

Keywords: recruitment, clinical research, adolescent research, pediatrician attitudes

\section{Introduction}

Recent federal guidelines calling for the inclusion of children in clinical research along with the United States Food and Drug Administration (FDA) incentives in children's drug development has led to a substantial increase in the number of clinical trials available within pediatric research. ${ }^{1-3}$ Although there are many practical problems associated with conducting clinical research, recruitment is often cited as the single most difficult problem to overcome in successfully implementing and completing a research protocol. ${ }^{4,5}$ The failure to recruit a sufficient number of participants can not only cause delays and increase costs associated with a research project, it can result in selection biases that limit the generalization of results ${ }^{6}$ and ultimately impede the introduction of new treatments in the field. ${ }^{7}$

Research investigating difficulties encountered in the recruitment process is relatively recent and prior studies have focused primarily on adult recruitment ${ }^{8-12}$ with few exceptions. ${ }^{13}$ This research has consistently documented the central role of a participant's physician in the recruitment and decision making process. ${ }^{11,14,15}$ Indeed, studies have now begun to look at the powerful effect of physician recommendation, either for or against participation in clinical research and several have suggested that physician preferences account for the majority of patients being entered or not entered into clinical research. ${ }^{15}$
Correspondence: Jeanne Dalen

2700 Yale Blvd. SE, Suite 200.Albuquerque, NM 87I06, USA

Tel $+1505842-8932$

Fax +1505 842-509।

Email jdalen@ori.org
Open Access Journal of Clinical Trials 2010:2 23-28

(c) 2010 Dalen et al, publisher and licensee Dove Medical Press Ltd. This is an Open Access article which permits unrestricted noncommercial use, provided the original work is properly cited. 
Communication between the patient and physician is, in many ways, a key element in recruitment for clinical research. For example, the Childhood Asthma Management Program (CAMP), a large multi-site pediatric study of mild to moderate asthma, reported that a factor critical for success in the recruitment effort was endorsement of research participation by the child's primary care provider. ${ }^{16}$ These investigators reported that even though a letter from the primary care provider had little impact, discussion between the physician and family during an office visit was very influential in the enrollment decision. This is consistent with other research which has shown that participants will rarely enroll in clinical research unless their physician actively recommends the trial. ${ }^{6,15}$

Physician enrollment recommendations have been shown to be an effective way of encouraging research participation in pediatric clinical research. ${ }^{13}$ However, the reluctance of physicians to offer patients a chance to participate in research is widespread. ${ }^{8}$ Factors related to both physician and research characteristics can serve as significant barriers to referral participation with suggestions that barriers reported for both internists and pediatricians are virtually identical. ${ }^{13}$ Concern about potential harm to the doctor-patient relationship, inadequate support from investigators, time constraints, insufficient physician interest in the questions posed by the research, and apprehension about particular procedures involved in the protocol are a few examples of the many complex reasons physicians feel reluctant to participate in the referral process. ${ }^{7,17,18}$

As a consequence of these perceived barriers, physicians may waver on the decision to recommend patient participation in clinical research. Thus, understanding physicians' attitudes regarding patient participation in clinical research is essential. With the increasing recognition of physicians' role in research recruitment, it is important not only to identify barriers reducing involvement but also to explore the factors that motivate referral recommendations and consequently increase the likelihood of participation in clinical research. Absent currently from the literature, is an understanding of both the research characteristics and physician characteristics that might make physicians more motivated to participate in the recruitment process. The purpose of this exploratory study was to determine the factors that influence pediatricians' referrals to pediatric clinical research. Furthermore, the study explores the relationship between pediatrician characteristics and self-reported number of patients referred to clinical research.

\section{Method}

\section{Participants and procedures}

Participants were pediatricians and pediatric sub-specialists recruited from an academic pediatrics department of a university children's hospital, an institution where the majority of pediatric research in the community is conducted. Pediatricians were recruited as part of a study designed to explore differences and similarities in perceptions of risk and benefit to participating in asthma-related research from the perspective of adolescents, parents, and pediatricians. ${ }^{19}$ The study was reviewed and approved by the Health Sciences Center's Human Research Review Committee. Campus mail was utilized to send all children's hospital pediatricians $(n=74)$ a study packet, including a demographic questionnaire and the Reasons for Referral Measure (RRM). A two-week reminder card was sent in an effort to increase participation. All participants were compensated with a token gift.

\section{Measures}

\section{Demographic questionnaire}

Sixteen demographic questions concerning age, ethnicity, and several specific practice questions (eg, year graduated from medical school), type of practice, description of clinical work, percentage of time spent in research activities, and their self-reported numbers of patients referred to clinical research studies in the past were included.

\section{Reasons for referral measure (RRM)}

A 10-item questionnaire was developed for this study to evaluate pediatrician perceptions of reasons influencing their decision to refer a patient to clinical research based on relevant domains identified in the literature. Using a 7-point Likert scale ( 1 = "not influential"- 7 = "very influential"), study participants rated the influence of various factors on their decision to refer a patient to clinical research (Table 2). There was an additional qualitative item entitled "other reasons" for participants to rank and explain any reason not already listed on the measure.

\section{Data analyses}

Data analysis occurred in three steps. First, in an effort to identify coherent subsets in the questionnaire data, a principal components factor analysis with varimax rotation was conducted on the RRM items. Scree plots and the eigenvalues were examined to determine the criterion for the number of factors. A factor loading cut-off point of 0.40 or higher was selected as the inclusion criterion for factor interpretation. ${ }^{20}$ Internal consistency of all factors consisting 
of two or more variables was assessed using the standardized Cronbach's coefficient alpha. ${ }^{21}$ As this analysis was exploratory in nature, an alpha value of 0.70 was preferred but 0.60 was defined as the lower limit of acceptability. ${ }^{22} \mathrm{Next}$, a within-subjects repeated measures multivariate analyses of variance (MANOVA) was conducted on the 4 factors identified in the principal components analysis to compare differences in importance among the reasons pediatricians refer patients. Finally, linear regression was used to explore the relationships between pediatrician characteristics (percentage of time spent in research and medical school graduating year) and self-reported number of patients referred to clinical research. The independent predictors were selected $a$ priori to allow for the investigation of the relative contributions of time in research activities and number of years in the training/practice.

\section{Results}

\section{Demographics}

Forty-three pediatricians participated in the study, yielding a return rate of $58 \%$ (Table 1). Ethnicity was reported as $68 \%$ non-Hispanic, 16\% Hispanic, 14\% Asian, and 2\% "other", which is representative of the population in the southwest United States from which the sample was collected. An average of $73 \%$ of these pediatricians' clinical work was spent with children aged 10 years or younger, $27 \%$ with adolescents (11-17 years old) with $13 \%$ of their clinical practice involving patients of all ages with asthma. The population served by these pediatricians was on Medicaid (66\%), third party insurance $(25 \%)$ or no insurance $(9 \%)$.

\section{RRM factor analysis}

RRM data from the 43 pediatricians was entered into a principal components analysis with varimax rotation using
SPSS v.14. Analysis was limited to 10 items of the RRM measure as there were no responses to the qualitative item entitled "other reasons." The analysis extracted four factors with eigenvalues exceeding 1.0 and accounting for $72 \%$ of the variance. The Cronbach's alpha for each of the four factors was 0.60 or higher. Factor loadings as well as percentage of variance are shown in Table 2. The first factor was comprised of contextual factors $(\mathrm{CF})$ of the research, including reputation of the researchers, sources of funding, and participant characteristics. The second factor represented the scientific merit (SM) of the research, including future benefit and importance of the research question. A third factor represented the perceived participant benefit (PB) resulting from the research, such as the direct and indirect medical benefit. A fourth factor comprised of a single questionnaire item, denoted compensation to self or program for referrals (PC). Though factors comprised of single items could potentially have poor reliability, ${ }^{23}$ it was not excluded from subsequent analyses due to its significance in driving referral behavior.

\section{Assessing differences between RRM factors}

To compare differences in reasons pediatricians refer patients, mean scores were constructed for the four factors and within-subjects repeated measures multivariate analysis of variance (MANOVA) was conducted. Each factor (CF, SM, $\mathrm{PB}$, and PC) was treated as a dependent variable. Differences among the influences for pediatrician referral to research were observed $(p=0.000)$, (partial eta squared $=0.81)$. The most influential consideration for referral was the scientific merit of the study (mean $=5.7)$, followed by the factor comprising patient benefit $($ mean $=4.9)$. Contextual factors and physician compensation were identified as the

Table I Pediatrician demographics $(n=43)$

\begin{tabular}{|c|c|c|c|}
\hline Mean age (range 30-74) & 46.7 & History of referrals (\%) & \\
\hline Gender (\%) & & None & 9.3 \\
\hline Male & 41.9 & I-5 referrals & 7.0 \\
\hline \multirow[t]{3}{*}{ Female } & 58.1 & $6-10$ referrals & 11.6 \\
\hline & & I I-20 referrals & 4.7 \\
\hline & & $>20$ referrals & 60.5 \\
\hline Mean years spent in practice (range 8-45) & 19.5 & No information given & 7.0 \\
\hline Medical specialty (\%) & & Time spent in research (\%) & \\
\hline Pediatric generalist & 46.5 & $<10 \%$ & 41.9 \\
\hline \multirow[t]{2}{*}{ Pediatric subspecialty } & 53.5 & $10 \%-25 \%$ devoted to research & 37 \\
\hline & & $>25 \%$ devoted to research & 21 \\
\hline
\end{tabular}


Table 2 Factor loadings of 10 reasons to refer patients to pediatric research

\begin{tabular}{|c|c|c|c|c|c|c|}
\hline Reasons for referral measure items & $\begin{array}{l}\text { Contextual } \\
\text { factors }\end{array}$ & $\begin{array}{l}\text { Scientific } \\
\text { merit }\end{array}$ & $\begin{array}{l}\text { Patient } \\
\text { benefit }\end{array}$ & $\begin{array}{l}\text { Physician } \\
\text { comp }\end{array}$ & $\begin{array}{l}\% \text { of } \\
\text { variance }\end{array}$ & $\begin{array}{l}\text { Cumulative } \\
\% \text { of } \\
\text { variance }\end{array}$ \\
\hline \multicolumn{7}{|l|}{ Contextual factors items } \\
\hline I. Patient likely to be cooperative & 0.7 I & -0.07 & 0.26 & 0.22 & & \\
\hline 2. Reputation of researchers & 0.87 & 0.05 & -0.02 & -0.13 & & \\
\hline 3. Institutional support for research & 0.66 & 0.57 & -0.22 & 0.23 & & \\
\hline 4. Source of funding for research (eg, grant/drug company) & 0.51 & -0.25 & 0.23 & -0.67 & 25.77 & 25.77 \\
\hline \multicolumn{7}{|l|}{ Scientific merit items } \\
\hline 5. Important-interesting research question & -0.09 & 0.85 & 0.14 & -0.15 & & \\
\hline 6. Likelihood of future benefit to patients not in the research & 0.05 & 0.91 & 0.11 & 0.09 & 18.81 & 44.58 \\
\hline \multicolumn{7}{|l|}{ Patient benefit items } \\
\hline 7. Opportunity for patient's direct medical benefit & -0.12 & 0.25 & 0.64 & 0.27 & & \\
\hline 8. Opportunity for indirect medical benefit to patient & 0.07 & -0.11 & 0.86 & -0.19 & & \\
\hline 9. Opportunity for other benefit to patient (eg, money, evaluations) & 0.33 & 0.19 & 0.70 & 0.14 & 15.86 & 60.45 \\
\hline \multicolumn{7}{|l|}{ Physician compensation items } \\
\hline 10. Compensation to you or your program for referrals & 0.05 & -0.05 & 0.11 & 0.88 & 11.21 & 71.66 \\
\hline
\end{tabular}

least important reasons pediatricians refer patients to research ( mean $=4.4$; mean $=2.1$, respectively $)$.

\section{Physician characteristics and referrals to clinical research}

Two analyses were conducted to examine the relationship between pediatrician characteristics and self-reported number of patients referred to clinical research. Linear regression was conducted using the percentage of time spent in research and graduating year from medical school and actual number of referrals made as the dependent variable. Results showed a significant relationship between the number of referrals made and percentage of time spent in research $\left(\mathrm{R}^{2}=0.18\right.$, $\mathrm{p}=0.008)$, but not graduating year from medical school $(\mathrm{p}=0.05)$ indicating that number of referrals to clinical research was affected more by the respondents' time in research activities rather than as a function of number of years in the training/practice.

\section{Discussion}

The purpose of this exploratory study was to examine factors pediatricians consider most influential when choosing to refer children and families to clinical research and to examine relationships between pediatrician characteristics and self-reported number of referrals to clinical research. Four key factors influencing pediatricians' decision to refer patients to clinical research were revealed: contextual factors, the scientific merit of the study, participant benefit, and physician compensation. Based upon mean scores, the most influential consideration for referral was the scientific merit of the study, including the importance of the research question and likelihood of future benefit to patients not currently involved in the research. Future benefit in this context related to the overall advancement of science. In addition, immediate patient benefit, described as either direct (specialized treatment for the child's medical condition, free medicine) or indirect (financial compensation) benefit was also considered important for referral. Of lesser importance, as compared to scientific merit and patient benefit, was physician compensation resulting from referrals and contextual factors such as the source of funding for the research. Though pediatricians in this study reported that these factors did not play as significant a role in their decision to refer, investigators must still be vigilant about minimizing the possibility for coercion and undue influence. Although these were University-based pediatricians and pediatric sub-specialists who may weigh the importance of biomedical research differently than pediatricians in the general community, these findings are consistent with reports by other investigators demonstrating that an interesting research question and the perception of some medical benefit is essential in motivating physician referral for research participation. ${ }^{5,8}$

A key implication of these findings is that pediatricians evaluate both the scientific merit and benefit components of a research study when suggesting research participation to their patients. Thus, emphasizing both the usefulness and importance of the research question and its contribution to advancements in the field may help increase referrals from 
pediatricians. Additionally, investigators may consider ways of designing studies that provide obvious patient benefit as part of the research protocol. For example, offering medical evaluations that offer diagnoses, providing evaluations of symptom severity, presenting recommendations for further treatment, regularly monitoring symptoms, and conducting specialized tests that might not be available in general clinics are all examples of patient benefits that might prompt pediatricians to recommend research participation to their patients.

However, the inclusion of additional patient benefit components to clinical studies may potentially blur the boundaries between research and standard clinical care for the patient, therefore "therapeutic misconception" is a critical issue to address. Therapeutic misconception refers to the tendency of research participants to confuse the design and conduct of research with personalized medical care. ${ }^{24}$ Though the focus of this article is on the potential utility of increasing patient benefit in order to increase referrals, informative discussions on behalf of the pediatrician and the investigator must focus on risks of the study as well.

Our findings also suggest that pediatrician referral behavior is, to some extent, reliant on specific characteristics of the individual pediatricians. For example, we found that those pediatricians who devote more time to research tend to refer more patients to clinical research. These results are consistent with findings showing a strong correlation between research experience and physician attitudes to clinical research trials; physicians with research experience perceived the most benefits for trial participation. ${ }^{25}$

There are several reasons why pediatricians spending more time in their own research activities may refer more patients to clinical research studies. They may be more invested in and supportive of research in general and since a larger proportion of their own time is spent in research they may be generally more familiar with the research process and value the potential significance of research studies for which they are asked to refer patients. In addition, they may have a clearer understanding of a research protocol's risk and benefit profile. This can assist in minimizing misinterpretations and misgivings regarding procedures, which have been implicated as potential barriers to recruitment. ${ }^{4}$ Lastly, pediatricians spending more time in their own research activities may be more sympathetic to the difficulties inherent to recruitment of subjects and therefore might be more willing to make referrals and assist in the process for other researchers.

By contrast, those pediatricians who devote less time to research may lack a connection to research, creating potential barriers to recruitment. This may necessitate that investigators work harder to engage these pediatricians in the research process. Investigators ought not to presume physicians' level of comfort or expertise with research, but instead take time to educate pediatricians on the research question of interest and on aspects of the research protocol that provide beneficial services to participants. This emphasis on education could assist in creating an understanding and acceptance of research that may help to facilitate the referral process.

Together, these findings have implications for increasing referral participation in pediatric research. First, investigators could potentially increase referring interest by emphasizing components of the clinical research study that directly address the worth of the science. Second, research suggests that pediatricians struggle with divided loyalties, expressing concern about the potential difficulties of balancing the needs of their patients while furthering science. ${ }^{12}$ Therefore, designing studies with clinical relevance and obvious patient benefit can help bridge that gap by appealing to pediatricians' desire to advance science while simultaneously helping to serve and care for their patients. More attention placed on enlisting the help of those pediatricians who spend time in their own research activities may substantially assist in recruitment. Finally, those pediatricians who devote less time to their own research activities may also be less familiar with research in general. Investigators may find it useful to spend time familiarizing these physicians with research protocol in order to increase referral participation.

There were several limitations to this study. Participants were restricted to pediatricians practicing within the same university research hospital so it remains unknown how pediatricians in non-university clinics may perceive the support of research recruitment. In addition, the response rate was relatively low at $58 \%$ therefore the characteristics of those who did not respond to the study might have been significantly different from those who did respond. Future research conducted on a larger and more varied sample would assist in determining whether these initial findings hold true. The RRM was developed specifically for this study and should not be considered a standardized measure since its psychometric properties would require further analyses. In addition, the principal components analysis was exploratory in nature and included a minimal number of items. However, with four distinct factors accounting for a total of $72 \%$ of the variance explained, some conservative conclusions can be drawn from this study.

This study has highlighted attitudes held by pediatricians that have implications for researchers wanting to elicit 
the help of pediatricians in the referral process for clinical research. Recruitment seeking representative participation can be arduous and time-consuming, thus investigators must find ways to improve their access to populations of suitable patients. Research has shown that an important avenue of successful recruiting is through primary care providers. Findings from this study suggest that the importance of the research question and perception of both present and future benefit to participants are among the most important considerations for pediatricians when deciding to refer a patient to research. In addition, characteristics related to the pediatricians' practice and the time they spend in their own research activities appears to influence referral behavior. Attention to these factors in the development of future studies may enhance pediatrician motivation and incentive to refer patients to clinical research.

\section{Acknowledgments}

Funding from the National Heart Lung and Blood Institute of the National Institutes of Health R01 HL64677.

\section{Disclosures}

The authors have no conflicts of interest that could impact the conduct or presentation of this study.

\section{References}

1. National Institutes of Health. NIH Policy and Guidelines on the Inclusion of Children as Participants in Research Involving Human Subjects. Washington DC: Department of Health and Human Services; 1991. Available from http://www.nih.gov/grants/guide/notice-files/not98-024. html Retrieved February 16, 2000.

2. Kauffman RE. Clinical trials in children: problems and pitfalls. Paediatr Drugs. 2000;2(6):411-418.

3. Botstein P. Why FDA is encouraging drug testing in children: FDA Consumer Special Report. Rockville MD: US Food, and Drug Administration; 1995.

4. Walson PD. Patient recruitment: US perspective. Pediatrics. 1999; 104(3 Pt 2):619-622.

5. Hoppu K. Patient recruitment: European perspective. Pediatrics. 1999;104(3 Pt 2):623-626.

6. Kinney AY, Richards C, Vernon SW, Vogel VG. The effect of physician recommendation on enrollment in the Breast Cancer Chemoprevention Trial. Prev Med. 1998;27(5 Pt 1):713-719.

7. Fallowfield L, Ratcliffe D, Souhami R. Clinicians' attitudes to clinical trials of cancer therapy. Eur J Cancer. 1997;33(13):2221-2229.
8. Ross S, Grant A, Counsell C, Gillespie W, Russell I, Prescott R. Barriers to participation in randomised controlled trials: a systematic review. J Clin Epidemiol. 1999;52(12):1143-1156.

9. Taylor KM, Feldstein ML, Skeel RT, Pandya KJ, Ng P, Carbone PP. Fundamental dilemmas of the randomized clinical trial process: results of a survey of the 1,737 Eastern Cooperative Oncology Group investigators. J Clin Oncol. 1994;12(9):1796-1805.

10. Taylor KM, Margolese RG, Soskolne CL. Physicians' reasons for not entering eligible patients in a randomized clinical trial of surgery for breast cancer. N Engl J Med. 1984;310(21):1363-1367.

11. Kaas R, Hart AA, Rutgers EJ. The impact of the physician on the accrual to randomized clinical trials in patients with primary operable breast cancer. Breast. 2005;14(4):310-316.

12. Ellis PM. Attitudes towards and participation in randomised clinical trials in oncology: a review of the literature. Ann Oncol. 2000;11(8): 939-945.

13. Caldwell PH, Butow PN, Craig JC. Pediatricians' attitudes toward randomized controlled trials involving children. $J$ Pediatr. 2002;141(6):798-803.

14. Brody JL, Scherer DG, Annett RD, Turner C, Dalen J. Family and physician influence on asthma research-participation decisions for adolescents: The effects of adolescent gender and research risk. Pediatrics. 2006;118:356-362.

15. Siminoff LA, Zhang A, Colabianchi N, Sturm CM, Shen Q. Factors that predict the referral of breast cancer patients onto clinical trials by their surgeons and medical oncologists. J Clin Oncol. 2000;18(6): 1203-1211.

16. Recruitment of participants in the childhood Asthma Management Program (CAMP). I. Description of methods: Childhood Asthma Management Program Research Group. J Asthma. 1999;36(3): 213-216.

17. Melisko ME, Hassin F, Metzroth L, et al. Patient and physician attitudes toward breast cancer clinical trials: developing interventions based on understanding barriers. Clin Breast Cancer. 2005;6(1):45-54.

18. Hinshaw SP, Hoagwood K, Jensen PS, et al. AACAP 2001 research forum: challenges and recommendations regarding recruitment and retention of participants in research investigations. J Am Acad Child Adolesc Psychiatry. 2004;43(8):1037-1045.

19. Annett RD, Brody JL, Scherer DG, Perkett EA. Perception of risk associated with asthma research procedures among adolescents, parents, and pediatricians. J Allergy Clin Immunol. 2004;114(5):1138-1145.

20. Hatcher L. A Step-by-Step Approach to Using the SAS System for Factor Analysis and Structural Equation Modeling. Cary, NC: SAS Institute Inc.; 1994.

21. Cronbach L. Coefficient alpha and the internal structure of tests. Psychometricka. 1951;16:297-334.

22. Hair J, Anderson RE, Tatham RL, et al. Multivariate Data Analysis. 5th ed. Upper Saddle River, NJ: Prentice-Hall; 1998.

23. Tabachnick BG, Fidell LS. Using Multivariate Statistics. 4th ed. Needham Heights, MA: Allyn and Bacon; 2001.

24. Appelbaum PS, Lidz CW, Grisso T. Therapeutic misconception in clinical research: frequency and risk factors. IRB. 2004;26(2):1-8.

25. Caldwell PH, Craig JC, Butow PN. Barriers to Australian physicians' and paediatricians' involvement in randomised controlled trials. Med J Aust. 2005;182(2):59-65.
Open Access Journal of Clinical Trials

\section{Publish your work in this journal}

The Open Access Journal of Clinical Trials is an international, peerreviewed, open access journal publishing original research, reports, editorials, reviews and commentaries on all aspects of clinical trial design, management, legal, ethical and regulatory issues, case record form design, data collection, quality assurance and data auditing

\section{Dovepress}

methodologies. The manuscript management system is completely online and includes a very quick and fair peer-review system, which is all easy to use. Visit http://www.dovepress.com/testimonials.php to read real quotes from published authors. 